\title{
PUCKERING INSTABILITY PHENOMENA IN THE HEMISPHERICAL CUP TEST
}

\author{
Nicolas TRIANTAFYLLIDIS \\ Department of Aerospace Engineering, The University of Michigan, \\ Ann Arbor, Michigan 48109-2140, U.S.A.
}

(Received 18 October 1983 ; in revised form 14 June 1984)

\begin{abstract}
THE PROBLEM investigated here is the plastic bifurcation of an initially flat circular plate held frictionlessly between a blankholder and a die and deformed by a spherically shaped punch.

In view of the large deviations of the prebifurcation solution from proportional loading, a recently developed phenomenological corner theory has been employed and an appropriate bifurcation criterion has been developed. The effects of geometry and material properties on the onset of the (nonaxisymmetric) plastic instability have been investigated using a numerical solution of the resulting equations based on the finite element method.
\end{abstract}

\section{INTRODUCTION}

AN INTERESTING plastic instability phenomenon, which, in spite of its practical importance in sheet metal forming applications, has received very little attention so far (to the best of the author's knowledge) is the so-called "puckering" phenomenon. The earliest reference to this problem in the literature appears to be in the book by DEvons (1941) where puckering is defined as the waviness formed in that part of the wall of a drawn shape that has already passed over the radius of the die (as opposed to wrinkling which is the term used for the formation of corrugations in the part of the blank which has not yet passed over the radius). A typical example of puckers in the wall of an almost hemispherical mild steel cup is shown in Fig. 1.

The specific problem to be investigated here is the plastic bifurcation of an initially flat circular plate held frictionlessly between a blankholder and a die and deformed by a spherically shaped punch as shown in Fig. 2. Since material can flow inwards from the outer flange to the cup's wall, compressive hoop stresses are developed at the contact free part of the shell, which, upon attaining a critical level, induce a nonaxisymmetric bifurcation. As noted by Devons (1941) and subsequently reemphasized by SHILKRUT (1983), the less the friction on the flange the more prone the structure is to buckling, in view of the developed higher compressive hoop stresses. Hence in order to examine the most unfavorable situation, the contact between the flange and the blankholder/die assembly is assumed perfectly smooth. For simplicity, the punch is also taken to be in frictionless contact with the shell.

In addition to practical importance, the problem at hand has the following theoretically interesting feature. In view of its particular geometry, severe deviations 


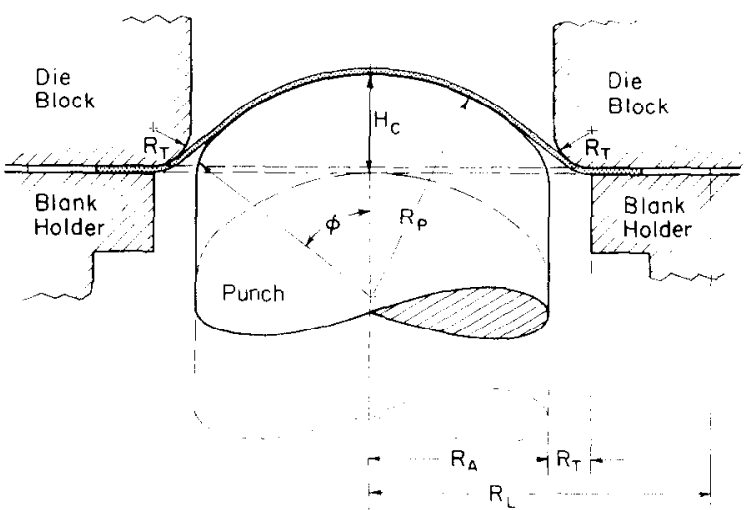

FIG. 2. Schematic drawing of the punch test. (- - -) initial stress free configuration; (- - final configuration.

from proportional loading exist in the (axisymmetric) prebifurcation solution. These deviations are the highest in exactly that part of the shell where the bifurcation eigenmode's amplitudes are expected to be maximal, i.e. in the contact free part of the structure. For reasonable plasticity models, the stability analysis of HiLL (1958) requires modifications, in view of the violation of the total loading condition in the prebifurcation state, and thus a suitable stability criterion has to be developed. Following some recent work by TRIANTAFYLLIDIS (1983), an appropriate bifurcation functional (similar to that of HILL (1958) but using the current incremental moduli instead of the hypoelastic comparison ones) is employed in order to provide a lower bound for the first critical load.

Due to the anticipated large deviations from proportional loading in the prebifurcation solution, a phenomenological corner theory of plasticity due to CHRISTOFFERSEN and HuTCHINSON (1979), capable of modeling the gradual stiffening of the incremental moduli as a material's loading path drifts away from proportional loading, is adopted. Classical finite strain generalizations of the $J_{2}$ deformation and the $J_{2}$ flow theories of plasticity can be obtained as special subcases of the aforementioned theory, by suitably modifying the vertex characteristics of the model.

The predictions of the deformation theory for the onset of puckering are compared and contrasted with results from corner type models all sharing the same uniaxial stress-strain curve and plastic anisotropy but having different vertex characteristics. In addition, the effects of punch curvature, drawing ratio (i.e. the ratio of the initial plate radius over the die aperture radius) initial plate thickness as well as material properties are also investigated (in particular, strain hardening and transverse plastic anisotropy). The effect of these parameters on the shape of the critical eigenmode, i.e. the number of puckers at bifurcation, is also explored.

\section{Prebifurcation Solution}

Consider an initially flat circular disc of uniform thick ness $h$ and radius $R_{L}$. The plate is held between a die block of a perture radius $R_{A}$ and a blankholder of aperture radius 


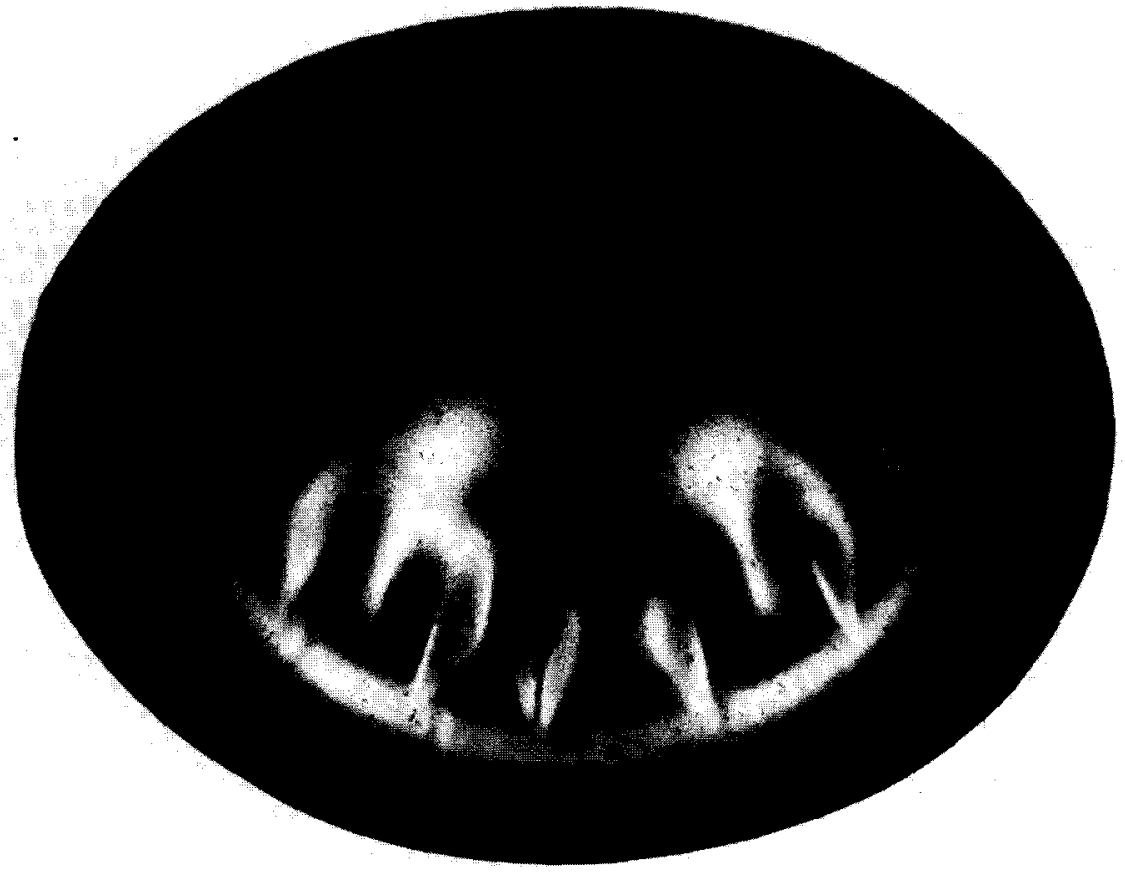

i. 1. Puckers in a mild steel hemispherical deep drawn cup. Courtesy of Dr. S. K. Samanta, Ford Motor Co., S.R.L., Dearborn, MI. 
$R_{A}+R_{T}$ where $R_{T}$ is the cross sectional radius of the die's throat as depicted in Fig. 2. A punch with a cylindrical base of radius $R_{A}$, carrying a spherical head of radius $R_{p}$, pushes the plate from its initial (flat) stress free configuration, shown in dotted lines in Fig. 2, into a deformed shape, indicated by solid lines on the same figure. Moreover $H$ denotes the total punch displacement from the unloaded configuration while the "punch angle", defined to be $\phi=\sin ^{-1}\left(R_{A} / R_{P}\right)$, is indicative of the "sharpness" of the punch; for $\phi=\pi / 2$ complete hemispherical cups can be drawn (at least in principle) while for lesser values of the angle $\phi$, spherical sector cups of weaker curvatures can be formed.

The contact between the plate and the punch as well as the contact between the plate and the die/blankholder assembly is considered to be frictionless. In addition it will be assumed that the disc's thickness is considerably smaller than the minimum possible radius of curvature of the resulting deformed shell, i.e. $h \ll R_{T}$ in order to justify the use of the membrane approximation for the prebifurcation solution of the problem. This assumption simplifies considerably not only the prebifurcation analysis but even more so the formulas for the bifurcation functional, as will be seen in the next section.

A full Lagrangian description for the equilibrium equations of the axisymmetric membrane is adopted here with the initial stress free configuration taken to be the reference one. If $u$ and $w$ are the horizontal and vertical displacements respectively of a material point initially at a distance $r$ from the plate's center, the internal virtual work can be written as $\dagger$

$$
\text { I.V.W. }=\int_{0}^{R_{L}}\left[(r+u) N_{r} \delta \varepsilon_{r}+\lambda_{r} r N_{\theta} \delta \varepsilon_{\theta}\right] \mathrm{d} r,
$$

where $N_{r}$ and $N_{\theta}$ are the current radial and hoop force resultants respectively given in terms of the radial and hoop Cauchy stresses $\sigma_{r}, \sigma_{\theta}$ and the current plate thickness $\bar{h}$ by

$$
N_{r}=\bar{h} \sigma_{r}, \quad N_{\theta}=\bar{h} \sigma_{\theta},
$$

while the strain measures $\varepsilon_{r}$ and $\varepsilon_{\theta}$, work conjugate to the stress measures $(r+u) N_{r}$ and $\lambda_{r} r N_{\theta}$ respectively, are

$$
\varepsilon_{r}=\lambda_{r}-1, \quad \varepsilon_{\theta}=\lambda_{\theta}-1 .
$$

Using simple geometrical considerations, the radial and circumferential stretch ratios $\lambda_{r}$ and $\lambda_{\theta}$ can be expressed in terms of the displacements $u$ and $w$ as follows

$$
\lambda_{r}=\left((1+\mathrm{d} u / \mathrm{d} r)^{2}+(\mathrm{d} w / \mathrm{d} r)^{2}\right)^{1 / 2}, \quad \lambda_{\theta}=\bar{r} / r=1+u / r,
$$

with $\bar{r}$ the current distance of the material point $r$ from the symmetry axis.

The external virtual work due to the frictionless contact between the punch and the membrane is taken to be

$$
\text { E.V.W. } ._{1}=-\delta\left[\frac{k}{2} \int_{0}^{R_{L}}\left(d_{1}-R_{P}\right)^{2} r \mathrm{H}\left(R_{P}-d_{1}\right) \mathrm{d} r\right],
$$

where $d_{1}$, the distance between a point with material coordinate $r$ and the center of the

† The expressions given for the I.V.W. and the E.V.W. are valid for a one radian sector of the plate. To find the I.V.W. and E.V.W. for the entire plate one has to multiply (2.1), (2.5) and (2.7) by $2 \pi$. 
punch's spherical head, is given by

$$
d_{1}=\left((r+u)^{2}+\left(w+R_{p}-H\right)^{2}\right)^{1 / 2} .
$$

The additional term for the external virtual work, due to the contact between the membrane and the die throat, constructed in the same fashion as (2.5), is

$$
\text { E.V.W.2 }=-\delta\left[\frac{k}{2} \int_{0}^{R_{L}}\left(d_{2}-R_{T}\right)^{2} r \mathrm{H}\left(R_{T}-d_{2}\right) \mathrm{d} r\right]
$$

where the analogously defined distance $d_{2}$ in this case assumes the form

$$
d_{2}=\left(\left(R_{A}+R_{T}-r-u\right)^{2}+\left(R_{T}-w\right)^{2}\right)^{1 / 2} .
$$

Note that in (2.5) and (2.7) H(x) designates the Heaviside function (defined as $\mathrm{H}(x)=0$ for $x<0, \mathbf{H}(x)=1$ for $x \geqslant 0)$. The above assumption for the external virtual work terms corresponds to a Winkler type foundation, for the punch and die surfaces, where the normal force per unit (reference) area is linearly proportional to the penetration depth $\left(d_{1}-R_{P}\right.$ or $\left.d_{2}-R_{T}\right)$ with coefficient of proportionality $k$.

For adequately high values of the "foundation constant" $k$ the punch and die surfaces will behave almost rigidly. The advantage of this type of formulation, usually called a penalty type of formulation in the literature, apart from its realistic physical interpretation (in reality no contact can ever be perfectly rigid; there is always a small amount of elastic deformation involved) simplifies considerably the resulting numerical calculations. Since the method decides automatically which part of the membrane is in contact with the rigid surfaces, it avoids complicated iteration schemes usually employed in similar analyses of sheet metal forming processes (see for example CHU (1980) for the axisymmetric case or WANG and BUDIANSKY (1978) for the general case).

The weak formulation of the equilibrium equation is obtained by equating the internal and external virtual work terms, namely

$$
\text { I.V.W. }=\text { E.V.W.1 }+ \text { E.V.W.2 } ._{2} \text { E.V.W. }
$$

and it will form the basis for the subsequent numerical analysis of the problem.

Next, attention is focused on the choice of a proper constitutive law. In this study the corner theory of CHRISTOFFERSEN and HUTCHINSON (1979) will be employed. This theory was introduced in order to develop an analytically tractable phenomenological theory of plasticity which would incorporate certain features exhibited by rate independent physical theories of plasticity. More specifically, in studies of the mechanical behavior of metal polycrystals using the single crystal slip model, the existence of corners at the current loading point on the yield surface has been predicted from the calculations (see for example Hill (1967), HUTCHnson (1970), LIN (1971); unfortunately, experimental evidence on the subject is ambiguous (HECKER, 1976)).

The significance of vertex formation for the bifurcation of solids in the plastic range has long been appreciated and deformation type theories of plasticity have successfully been used repeatedly in the past for plastic buckling calculations (for an extended discussion on the subject the interested reader is referred to HuTCHINSON (1973, 1974)). In the problem at hand, however, a simpler deformation type theory of plasticity (as a model for a vertex material) would be unacceptable even for the onsel of plastic 
buckling calculations, in view of the significant deviations of the structure from proportional loading in the prebifurcation branch of the solution. As will be seen in the numerical calculations to follow, in the unsupported part of the plate (between the punch and the die) where the bifurcation eigenmode's amplitude will be maximal, some material points might even undergo elastic unloading prior to the onset of plastic buckling. In such cases the corner theory of Christoffersen and Hutchinson is an attractive alternative and has been successfully used in the past on several applications (see for example TVergaARD, NeEdleman and Lo, 1981; Triantafyllidis, NeEdleman and TVergaARD, 1982; TVERGAard, 1982). An additional feature of this corner theory is the smooth dependence of the incremental moduli (or compliances) on the strain (or stress) rates which makes its use particularly attractive in bifurcation calculations where elastic unloading is present in part of the prebifurcation solution (see TVERGAARD (1982)), as it is the case at hand.

In the corner theory employed here the instantaneous moduli in the forward loading cone are chosen to be the moduli of a deformation theory of plasticity, appropriately modified for large strains, that takes into account the transverse anisotropy of the sheet. The aforementioned deformation theory was also used by TRIANTAFYLLIDIS and NEEDLEMAN (1980) in the wrinkling studies of the Swift cup test and the reader is referred therein for further details. For increascd deviations from proportional loading the moduli stiffen monotonically until they reach the values of the linearly elastic moduli for stress rates directed inside the loading cone.

For the deformation theory model of an anisotropic elasto-plastic material, the plastic compliance tensor $\mathbf{C}$ (relating the plastic part of the strain rate tensor to the Jaumann rate of the Kirchhoff stress tensor) is found to be

$$
C_{i j k l}=\left(\frac{1}{E_{s}}-\frac{1}{E}\right) \frac{\partial^{2} \phi}{\partial \tau^{i j} \partial \tau^{k l}}+\frac{1}{\tau_{e}^{2}}\left(\frac{1}{E_{t}}-\frac{1}{E_{s}}\right) \frac{\partial \phi}{\partial \tau^{i j}} \frac{\partial \phi}{\partial \tau^{k l}},
$$

where $\tau$ is the Kirchhoff stress tensor $\ddagger$ and $\tau_{e}$ the equivalent stress for the material given by the following expression

$$
\begin{aligned}
\tau_{e}^{2}= & 2 \phi \equiv \\
& \frac{\left(\tau^{<11>}-\tau^{<33>}\right)^{2}+\left(\tau^{<22>}-\tau^{<33>}\right)^{2}+R\left(\tau^{<11>}-\tau^{<22>}\right)^{2}+2(1+2 R)\left(\tau^{<12>}\right)^{2}}{1+R}
\end{aligned}
$$

in the case of a transversely plastically anisotopic material, with anisotropy ratio $R$, under plane stress conditions. The uniaxial behavior of the material will be taken to be a piecewise power law in which case the secant and tangent moduli $E_{s}$ and $E_{t}$ will be

\footnotetext{
† Here and subsequently, Latin indices range from 1 to 3 while Greek ones range from 1 to 2 . The standard summation conventions of the tensor calculus are also adopted.

¥The constitutive equations employed here are formulated in terms of the Kirchhoff instead of the Cauchy stresses in view of the resulting symmetries in the incremental moduli. The difference of the two stress measures, due to elastic compressibility, is negligible in plasticity problems.
} 
respectively given by the expressions

$$
\begin{gathered}
E_{s}= \begin{cases}E\left(\tau_{e} / \sigma_{y}\right)^{m} & \text { for } \sigma_{y} \leqslant \tau_{e}, \\
E & \text { for } \sigma_{y}>\tau_{e},\end{cases} \\
E_{t}= \begin{cases}E_{s} / m & \text { for } \sigma_{y} \leqslant \tau_{e}, \\
E & \text { for } \sigma_{y}>\tau_{e},\end{cases}
\end{gathered}
$$

where $E$ is the Young's modulus, $m$ the hardening exponent and $\sigma_{y}$ the yield stress of the uniaxial stress-strain curve. The forward loading cone axis, in stress rate space, is taken to be

$$
\lambda^{i j}=\tau^{i j} /\left(\tau^{m n} C_{m n r s} \tau^{r s}\right)^{1 / 2}
$$

An angular measure $\theta$ of the deviation of the stress rate direction relative to the above introduced axis is defined by

$$
\cos \theta=\left(\lambda^{i j} C_{i j k l}{ }^{\nabla}{ }^{k l}\right) /\left(\stackrel{\nabla}{\tau}^{m n} C_{m n r s}{ }^{\nabla r s}\right)^{1 / 2},
$$

where $\nabla$ is the Jaumann rate of the Kirchhoff stress. The stress rate potential at the vertex is

$$
W=\frac{1}{2} \bar{\tau}^{i j}\left(M_{i j k l}+f(\theta) C_{i j k l}\right)^{\frac{\nabla}{k l}},
$$

where $\mathbf{M}$ is the well known tensor of linear elastic compliances with Cartesian components

$$
M_{i j k l}=\frac{1+v}{E}\left[\frac{1}{2}\left(\delta_{i k} \delta_{j l}+\delta_{i l} \delta_{j k}\right)-\frac{v}{1+v} \delta_{i j} \delta_{k l}\right] \text {, }
$$

with $v$ denoting Poisson's ratio. The transition function $f(\theta)$ is taken to be

$$
f(\theta)= \begin{cases}1 & \text { for } 0 \leqslant \theta \leqslant \theta_{0} \\ \cos ^{2}\left[\frac{\pi}{2}\left(\frac{\theta-\theta_{0}}{\theta_{c}-\theta_{0}}\right)\right] & \text { for } \theta_{0} \leqslant \theta \leqslant \theta_{c} \\ 0 & \text { for } \theta_{c} \leqslant \theta \leqslant \pi\end{cases}
$$

where $\theta_{0}$ and $\theta_{c}$ satisfy $\theta_{0} \leqslant \theta_{c}-\pi / 2$. The strain rate tensor is then related to the stress rate by

$$
\dot{E}_{i j}=\frac{\partial W}{\partial \eta^{i j}}\left(=\frac{\partial^{2} W}{\partial \tau^{i j} \partial \tau^{\nabla^{k}}}{ }^{\nabla k l}\right)
$$

the second equality in parentheses being an obvious consequence of the homogeneity of degree two of $W$ in terms of $\frac{\nabla}{\tau}$. One can show that in this case (2.19) can always be inverted due to the convexity of $W \dagger$, in order to give the stress rate in terms of the strain rate. The tensor of incremental moduli $\mathbf{L}$ is the inverse of the compliance tensor $\partial^{2} W / \partial t \partial \nabla$. Denoting by $L^{<i j k l>}$ the physical components of $\mathbf{L}$ and using the plane 
stress approximation, the constitutive equations take the form

$$
\dot{\tau}^{<\alpha \beta\rangle}=\left[L^{<\alpha \beta \gamma \delta>}-\frac{L^{<\alpha \beta 33>} L^{<33 \gamma \delta>}}{L^{<333>}}\right] \dot{E}_{<\gamma \delta>},
$$

with the physical components of the strain rate tensor given by

$$
\dot{E}_{<11>}=\lambda_{r} / \lambda_{r}, \quad \dot{E}_{<22>}=\lambda_{\theta} / \lambda_{\theta}, \quad \dot{E}_{<33>}=\dot{\bar{h}} / \bar{h} .
$$

The constitutive equation for the material in conjunction with the rate form of the equilibrium equation (2.9) will furnish the prebifurcation solution of the problem via a f.e.m. technique, as will be described in the appropriate section.

\section{Bifurcation Analysis}

\subsection{General formulation}

As mentioned in the introduction, HiLl's (1958) theory for bifurcation and uniqueness requires modification for the problem at hand in view of the nonapplicability of the hypoelastic comparison solid concept due to the presence of strong deviations from proportional loading in the principal branch of the solution. Consequently, the appropriate bifurcation criterion for corner type theories of plasticity under quite general prebifurcation conditions will be outlined here. For more details on this subject the interested reader is referred to some recent work by TRIANTAFYLLIDIS (1983).

Consider a body occupying a volume $V$ and bounded by a surface $\partial V$ in the reference configuration. Material points are identified by a set of convected coordinates $x^{i}$ and a full Lagrangian formulation of the field equations is adopted. Moreover, a quantity surmounted by a dot ( $)$ denotes the corresponding derivative with respect to some monotonically increasing parameter, also termed the "time-like parameter", while a quantity followed by a comma (,) indicates covariant differentiation with respect to the reference metric.

The prebifurcation state of the body can be completely specified as a function of the time-like parameter, say $\lambda$, and all quantities associated with the principal branch of the solution are labeled by a superscript or subscript zero. The fundamental solution starts at $\lambda=0$ and is associated with monotonically increasing $\lambda$. In the specific application treated in this work, $\lambda$ is identified with the punch displacement parameter $H$.

Suppose that at some stage of deformation, characterized by $\lambda$, the displacements $\mathbf{u}(\hat{\lambda})$ the (Kirchhoff) stresses $\tau(\lambda)$, and any state variable that might be involved in the constitutive theory used, as well as their corresponding rates $\dot{\mathbf{u}}(\lambda), \dot{\tau}(\lambda)$, etc. are known and equal to the corresponding quantities evaluated on the principal branch. An increment $\Delta \dot{\lambda}$ in $\lambda$ produces the following displacement and stress increment in the aforementioned field quantities

$$
\begin{array}{ll}
\Delta \tau^{i j}=i_{0}^{i j} \Delta \lambda+\frac{1}{2} i^{i j}(\Delta \lambda)^{2}+\cdots ; & \Delta u_{k}=\dot{u}_{k}^{0} \Delta \lambda+\frac{1}{2} \ddot{u}_{k}(\Delta \lambda)^{2}+\cdots, \\
\Delta i^{i j}=\ddot{\tau}^{i j} \Delta \lambda+\frac{1}{2} i^{i j}(\Delta \lambda)^{2} \cdots ; & \Delta \dot{u}_{k}=\ddot{u}_{k} \Delta \lambda+\frac{1}{2} \cdot \ddot{u}_{k}(\Delta \lambda)^{2}+\cdots .
\end{array}
$$

If, at this $\lambda$, bifurcation is possible, the increment $\Delta \lambda$ produces two different solutions for the problem, namely $\left(\Delta u_{k}\right)^{\alpha},\left(\Delta \tau^{i j}\right)^{\alpha}$ and $\left(\Delta u_{k}\right)^{\beta},\left(\Delta \tau^{i j}\right)^{\beta}$. Introduce the following notation 
for the difference between two possible solutions $\alpha$ and $\beta$ in any field quantity:

$$
\tilde{C} \equiv \Delta()^{\alpha}-\Delta()^{\beta}\left(=()^{\alpha}-()^{\beta}\right) \text {. }
$$

Then, from the principle of virtual work, evaluated at $\lambda+\Delta \lambda$, and since in view of the assumptions $\tilde{u}_{k}(\lambda)=\dot{\tilde{u}}_{k}(\lambda)=\tilde{\tau}^{i j}(\lambda)=\dot{\tau}^{i j}(\lambda)=0$, the first nontrivial term (which involves ( ) quantities) obtained is the $(\Delta \lambda)^{2}$ term, namely

$$
0=\int_{\partial V}\left(\ddot{\tilde{T}}^{i} \ddot{\tilde{u}}_{i}\right) \mathrm{d} S=\int_{V}\left(\ddot{\tilde{\tau}}^{i j} \ddot{\tilde{E}}_{i j}+\tau_{0}^{i j} \ddot{\tilde{u}}_{, i}^{k} \ddot{\tilde{u}}_{k, j}\right) \mathrm{d} V,
$$

where

$$
\ddot{\tilde{E}}_{i j}=\frac{1}{2}\left(\ddot{\tilde{u}}_{i, j}+\ddot{\tilde{u}}_{j, i}\right)+\frac{1}{2}\left(\ddot{u}_{, i}^{k} \ddot{\tilde{u}}_{k, j}+\ddot{u}_{, j}^{k} \ddot{\tilde{u}}_{k, i}\right) \text {. }
$$

The surface term in (3.3) vanishes since, on the boundary, $\tilde{T}^{i}=0$ on that part of the surface where the traction vector $T^{i}$ is prescribed and $\tilde{u}_{i}=0$ on the part of the surface with given displacements. An additional relation between $\ddot{\tilde{\tau}}^{i j}$ and $\tilde{\tilde{E}}_{i j}$ will be provided from the constitutive law as follows.

By taking rates in (2.19) and subsequently applying the difference () operator one obtains (using also the homogeneity of degree two of $W$ with respect to $\frac{\bar{\tau}}{\tau}$ )

$$
\ddot{\tilde{E}}_{i j}=\left(\frac{\partial^{2} W}{\partial \nabla^{i j} \partial \tilde{\tau}^{k l}}\right)_{0}\left(\ddot{\tau}^{k l}+g_{0}^{k m} \tau_{0}^{l n} \ddot{\tilde{E}}_{m n}+g_{0}^{l m} \tau_{0}^{k n} \ddot{\tilde{E}}_{m n}\right) .
$$

The convexity of $W$ as a function of $\nabla^{i j}$ assures the invertibility of the positive definite fourth rank tensor $\partial^{2} W / \partial \tau \partial^{\nabla} \dagger$ as discussed in the previous section and thus (3.5) yields

with

$$
\ddot{\tau}^{i j}=\mathscr{L}_{0}^{i j k l} \ddot{\widetilde{E}}_{k l},
$$

$$
\mathscr{L}_{0}^{i j k l}=L_{0}^{i j k l}-\frac{1}{2}\left[g_{0}^{i k} \tau_{0}^{j l}+g_{0}^{i l} \tau_{0}^{j k}+g_{0}^{j l} \tau_{0}^{i k}+g_{0}^{j k} \tau_{0}^{i l}\right],
$$

where $g_{i j}$ are the contravariant components of the current metric tensor. It must be remarked here that the incremental moduli tensor $\mathbf{I}_{0}$ is constructed in such a fashion as to share the same indicial symmetries as its inverse $\left(\partial^{2} W / \partial \tau \tau \tau\right)_{0}$; consequently, the same symmetries will be inherited by $\mathscr{L}_{0}$, as a simple inspection of (3.6) shows. Substituting (3.6) into the right hand side of (3.3), the following bifurcation functional, quadratic in $\ddot{\tilde{\mathbf{u}}}$, is defined :

$$
F\left[\lambda, \ddot{\tilde{u}}_{i}\right]=\int_{V}\left[\mathscr{L}_{0}^{i j k l} \Delta \ddot{\tilde{E}}_{i j} \Delta \ddot{\widetilde{E}}_{k l}+\tau_{0}^{i j} \ddot{\tilde{u}}_{, i}^{k} \ddot{\tilde{u}}_{k, j}\right] \mathrm{d} V .
$$

Positive definiteness of $F$ for a given $\lambda$ excludes the possibility of bifurcation at this point. The assumption of uniqueness for the rates $\dot{\tilde{\mathbf{u}}}(\lambda), \dot{\tau}(\lambda)$, etc., required for consistency in the linearization process of the constitutive equations, is in no way of $\boldsymbol{t}$.

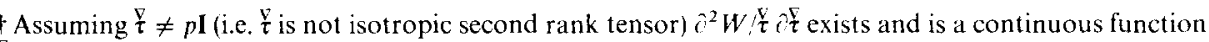


restrictive of the argument's generality for the following reason: in view of the material's rate independence, any adequately smooth change in the time-like parameter leaves the displacement, stress and internal variable fields in the solution of the problem unchanged. It is thus always possible to consider that at the point of interest, say $\lambda_{c}$, the new time-like parameter $\hat{\lambda}$ satisfies $\mathrm{d} \lambda / \mathrm{d} \hat{\lambda}=0$ but $\mathrm{d}^{2} \lambda /(\mathrm{d} \hat{\lambda})^{2} \neq 0$ and hence $\tilde{\mathbf{u}}\left(\lambda_{c}\right)=\mathrm{d} \tilde{\mathbf{u}}(\lambda) / \mathrm{d} \hat{\lambda}_{\mathrm{c}}=0$. Consequently, positive definiteness of $F$ for all $\lambda$ in an interval $\left(0, \lambda_{c}\right)$ assures uniqueness of the principal branch in that interval. Bifurcation becomes possible for the first time at this particular $\lambda=\lambda_{\mathrm{c}}$ for which the positive definiteness is lost. At this point, the corresponding eigenmode $\mathbf{u}^{(1)}$ satisfies

$$
F\left(\lambda_{c}, u_{i}^{(1)}\right)=0, \quad \delta F\left(\lambda_{c}, u_{i}^{(1)}\right)=0,
$$

where $u_{i}^{(1)}$ is subjected to the boundary conditions $u_{i}^{(1)}=0$ on the part of $\partial V$ with prescribed displacements.

It should be noted that the bifurcation functional $F$ used here differs from the classical one of HILL (1958) in that the incremental moduli are not those of the linear comparison solid but the actual ones evaluated at the principal branch of the solution. For prebifurcation paths satisfying total loading the two functionals coincide as expected. The functional (3.7) has also been used by TVERGAard (1982) to provide an upper bound for the critical load in his cylinder buckling problem while in this case, it is employed in the search for a lower bound for the first bifurcation.

\subsection{Problem formulation}

Here, for simplicity, the current configuration will be taken to coincide with the reference one. The convected coordinates to be used are $x^{1}=r, x^{2}=\theta, x^{3}=\tilde{z}$ where $\bar{z}$ is the current distance of a material point from the shell's reference surface (taken to be the deformed configuration of the middle surface in the stress free state) while $r, \theta$ are the polar coordinatest of a material point in the unloaded configuration of the aforementioned surface. In addition, the classical Kirchhoff-Love shell theory approximations are invoked. From the plane stress assumption $t^{i 3}=0$, one deduces

$$
\tilde{\tau}^{\alpha \beta}=\hat{L}_{0}^{\alpha \beta \gamma \ddot{z}} \ddot{E}_{y \delta}, \quad \ddot{\tau}^{i 3}=0,
$$

with the plane stress incremental moduli $\hat{\mathbf{L}}_{0}$ given in terms of $\mathscr{L}_{0}$ in (3.6) by

$$
\hat{L}_{0}^{\alpha \beta \gamma \delta}=\mathscr{L}_{0}^{\alpha \beta \gamma \delta}-\mathscr{L}_{0}^{\alpha \beta 33} \mathscr{L}_{0}^{33 \gamma \delta} / \mathscr{L}_{0}^{3333}
$$

Consequently, the bifurcation functional $F$ in (3.7) assumes the form

$$
F=\int_{V}\left[\hat{L}_{0}^{\alpha \beta \gamma \delta} \ddot{\tilde{u}}_{\alpha, \beta} \ddot{\tilde{u}}_{\gamma, \delta}+\tau_{0}^{\alpha \beta} \tilde{u}_{, \alpha}^{\gamma} \ddot{\tilde{u}}_{\gamma, \beta}+\tau_{0}^{\alpha \beta} \ddot{\tilde{u}}_{3, \alpha} \dot{\tilde{u}}_{3, \beta}\right] \mathrm{d} V .
$$

Note that the covariant differentiations appearing in (3.11) are to be considered with

\footnotetext{
$\dagger$ The polar coordinate $\theta$ is not to be confused with the angular measure $\theta$ of the deviation of the strain rate from proportional loading, introduced in (2.15).
} 
respect to the three dimensional metric. Further simplifications due to the axisymmetry of the reference configuration are possible and work as follows.

The covariant components of the current metric tensor for a material point $r, \theta, \bar{z}$ can be found from simple geometrical considerations to be

$$
\left.\begin{array}{c}
g_{11}^{0}=\left(\lambda_{r}\left(1+\bar{\kappa}_{r} \bar{z}\right)\right)^{2}, \quad g_{22}^{0}=\left(\bar{r}\left(1+\bar{\kappa}_{\theta} \bar{z}\right)\right)^{2}, \\
g_{33}^{0}=1, \quad g_{i j}^{0}=0 \text { for } i \neq j,
\end{array}\right\}
$$

where $\lambda_{r}$ is the stretch ratio in the radial direction of a point on the shell's reference surface given by $(2.4), \bar{\kappa}_{r}$ and $\bar{\kappa}_{\theta}$ are the principal curvatures of the same surface along the radial and tangential directions respectively while $\bar{\phi}$ is the angle formed between the outward normal to the aforementioned surface and the shell's axis. From elementary kinematics for the axisymmetric surface, one obtains

$$
\begin{gathered}
\sin \bar{\phi}=-(\mathrm{d} w / \mathrm{d} r) / \lambda_{r}, \quad \cos \bar{\phi}=(1+(\mathrm{d} u / \mathrm{d} r)) / \lambda_{r}, \\
\bar{\kappa}_{r}=\left((\mathrm{d} w / \mathrm{d} r)\left(\mathrm{d}^{2} u / \mathrm{d} r^{2}\right)-(1+\mathrm{d} u / \mathrm{d} r)\left(\mathrm{d}^{2} w / \mathrm{d} r^{2}\right)\right) / \lambda_{r}^{3}, \quad \bar{\kappa}_{\theta}=(\sin \bar{\phi}) / \bar{r}
\end{gathered}
$$

with $u(r), w(r)$ the radial horizontal and vertical displacements of the material point $(r, \theta, 0)$ of the (initially) middle surface from its initial flat position to its current configuration which is the shell's reference surface. Using (3.13) in conjunction with the standard definitions of covariant derivatives, the physical components of the $\ddot{\tilde{u}}_{i, j}$ tensor are found to be

$$
\begin{gathered}
\ddot{\tilde{u}}_{<1,1>}=\left[\frac{\partial \ddot{\tilde{u}}_{<1>}}{\partial r}+\lambda_{r} \bar{\kappa}_{r} \ddot{\tilde{u}}_{<3>}\right] /\left[\lambda_{r}\left(1+\bar{\kappa}_{r} \bar{z}\right)\right], \\
\ddot{\tilde{u}}_{<1,2>}=\left[\frac{\partial \ddot{\tilde{u}}_{<1>}}{\partial \theta}-\cos \bar{\phi} \ddot{\tilde{u}}_{<2>}\right] /\left[\vec{r}\left(1+\bar{\kappa}_{\theta} \bar{z}\right)\right], \\
\ddot{\tilde{u}}_{<2,1>}=\frac{\partial \ddot{\tilde{u}}_{<2>}}{\partial r} /\left[\lambda_{r}\left(1+\bar{\kappa}_{r} \bar{z}\right)\right], \\
\ddot{\tilde{u}}_{<2,2>}=\left[\frac{\partial \ddot{\tilde{u}}_{<2>}}{\partial \theta}+\cos \bar{\phi} \ddot{\tilde{u}}_{<1>}+\sin \phi \ddot{\tilde{u}}_{<3>}\right] /\left[\bar{r}\left(1+\bar{\kappa}_{\theta} z\right)\right], \\
\ddot{\tilde{u}}_{<3,1>}=\left[\frac{\partial \ddot{\tilde{u}}_{<3>}}{\partial r}-\lambda_{r} \bar{\kappa}_{r} \ddot{\tilde{u}}_{<1>}\right] /\left[\lambda_{r}\left(1+\bar{\kappa}_{r} \tilde{z}\right)\right], \\
\ddot{\tilde{u}}_{<3,2>}=\left[\frac{\partial \ddot{\tilde{u}}_{<3>}}{\partial \theta}-\sin \bar{\phi} \ddot{\tilde{u}}_{<2>}\right] /\left[\vec{r}\left(1+\bar{\kappa}_{\theta} \bar{z}\right)\right] .
\end{gathered}
$$

The normality part of the Kirchhoff-Love hypothesis is expressed as

$$
\ddot{\tilde{u}}_{i}\left(x^{1}, x^{2}, x^{3}\right)=a_{i}\left(x^{1}, x^{2}\right)+x^{3} \delta_{i}\left(x^{1}, x^{2}\right),
$$


with $\delta_{i}$ given in terms of the reference surface's velocity increments $a_{i}$ by

$$
\delta_{\alpha}=-\left(\frac{\partial a_{3}}{\partial x^{\alpha}}+B_{\alpha}^{\beta} a_{\beta}\right) ; \quad \delta_{3}=0 .
$$

Denoting $a_{<1>}=u, a_{<2>}=\bar{v}, a_{<3\rangle}=\bar{w}$ and employing (3.16), (3.17), the physical components of $\ddot{\tilde{u}}_{i}$ which enter (3.15) take the form

$$
\left.\begin{array}{c}
\ddot{\tilde{u}}_{<1>}=\left[\lambda_{r}\left(1+\overline{\kappa_{r}} \bar{z}\right) \bar{u}-\bar{z} \frac{\partial \bar{w}}{\partial r}\right] / \lambda_{r}, \\
\ddot{\tilde{u}}_{<2>}=\left(1+\bar{\kappa}_{\theta} \bar{z}\right) \bar{v}-\frac{\bar{z}}{\bar{r}} \frac{\partial \bar{w}}{\partial \theta}, \quad \ddot{\tilde{u}}_{<3>}=\bar{w} .
\end{array}\right\}
$$

Direct use of (3.18) in (3.15) and substitution of the resulting formulas into the bifurcation functional (3.11) results in an extremely cumbersome expression. Thus, at this stage, it is additionally assumed that the shell's minimum radius of curvature is much larger than its thickness, i.e. $\left|\bar{\kappa}_{r} \bar{z}\right| \ll 1,\left|\bar{\kappa}_{\theta} \bar{z}\right| \ll 1$ and that the prebifurcation state is a membrane state, i.e. the $\bar{z}$ (thickness) dependence of the incremental moduli as well as the stresses is negligible. Under these conditions the bifurcation functional $F$ assumes the form

$$
\begin{aligned}
F= & \int_{0}^{2 \pi} \int_{0}^{R_{L}}\left\{C_{11}\left[\left(\frac{\partial \bar{u}}{\partial r}+\lambda_{r} \bar{\kappa}_{r} \bar{w}\right)^{2}+\frac{\hbar^{2}}{12}\left(\frac{\partial}{\partial r}\left(\frac{1}{\lambda_{r}} \frac{\partial \bar{w}}{\partial r}\right)\right)^{2}\right]\right. \\
& +C_{22}\left[\left(\frac{\partial \bar{v}}{\partial \theta}+\bar{u} \cos \bar{\phi}+\bar{w} \sin \bar{\phi}\right)^{2}+\frac{h^{2}}{12}\left(\frac{1}{\bar{r}} \frac{\partial^{2} w}{\partial \theta^{2}}+\frac{\cos \Phi}{\lambda_{r}} \frac{\partial \bar{w}}{\partial r}\right)^{2}\right] \\
& +2 C_{12}\left[\left(\frac{\partial \bar{u}}{\partial r}+\lambda_{r} \bar{\kappa}_{r} \bar{w}\right)\left(\frac{\partial \bar{v}}{\partial \theta}+\bar{u} \cos \bar{\phi}+\bar{w} \sin \bar{\phi}\right)\right. \\
& \left.+\frac{\hbar^{2}}{12}\left(\frac{\partial}{\partial r}\left(\frac{1}{\lambda_{r}} \frac{\partial \bar{w}}{\partial r}\right)\right)\left(\frac{1}{\bar{r}} \frac{\partial^{2} \bar{w}}{\partial \theta^{2}}+\frac{\cos \bar{\phi}}{\lambda_{r}} \frac{\partial \bar{w}}{\partial r}\right)\right] \\
& +D_{11}\left[\left(\frac{\partial \bar{v}}{\partial r}\right)^{2}+\frac{\hbar^{2}}{12}\left(\frac{\partial}{\partial r}\left(\frac{1}{\bar{r}} \frac{\partial \bar{w}}{\partial \theta}\right)\right)^{2}\right]+D_{22}\left[\left(\frac{\partial \bar{u}}{\partial \theta}-\bar{v} \cos \phi\right)^{2}\right. \\
& \left.+\frac{h^{2}}{12}\left(\frac{1}{\lambda_{r}} \frac{\partial^{2} \bar{w}}{\partial r} \frac{\cos \bar{\phi}}{\bar{\phi}} \frac{\partial \bar{w}}{\partial \theta}\right)^{2}\right]+2 D_{12}\left[\left(\frac{\partial \bar{v}}{\partial r}\right)\left(\frac{\partial \bar{u}}{\partial \theta}-\bar{v} \cos \bar{\phi}\right)\right. \\
& \left.+\frac{h^{2}}{12}\left(\frac{\partial}{\partial r}\left(\frac{1}{\bar{r}} \frac{\partial \bar{w}}{\partial \theta}\right)\right)\left(\frac{1}{\lambda_{r}} \frac{\partial^{2} \bar{w}}{\partial r}-\frac{\cos \phi}{\bar{r}} \frac{\partial \bar{w}}{\partial \theta}\right)\right] \\
& \left.+N_{r}\left(\frac{\partial w}{\partial r} \frac{1}{\lambda_{r}}\right)^{2}+N_{\theta}\left(\frac{1}{\bar{r}} \frac{\partial w}{\partial \theta}\right)^{2}\right\} \bar{r} \lambda_{r} \mathrm{~d} r \mathrm{~d} \theta,
\end{aligned}
$$

$\dagger B_{\beta}^{\alpha}$ is the second fundamental form of the reference surface. Here $B_{1}^{1}=\bar{\kappa}_{r}, B_{2}^{2}=\bar{\kappa}_{\dot{\theta}}$. 
where the coefficients $C_{\alpha \beta}, D_{\alpha \beta}$ are defined as follows

$$
\begin{aligned}
& C_{11}=\bar{h} \frac{\hat{L}_{<1111>}^{0}+\tau_{<11>}^{0}}{\lambda_{r}^{2}}, \\
& C_{12}=\bar{h} \frac{\hat{L}_{<1122>}^{0}}{\lambda_{r} \bar{r}}, \\
& C_{22}=\bar{h} \frac{\hat{L}_{<222>}^{0}+\tau_{<22>}^{0}}{\bar{r}^{2}}, \\
& D_{11}=\bar{h} \frac{\hat{L}_{<1212>}^{0}+\tau_{<11>}^{0}}{\lambda_{r}^{2}}, \\
& D_{12}=\bar{h} \frac{\hat{L}_{<1212>}^{0},}{\lambda_{r} \bar{r}} \\
& D_{22}=\hbar \frac{\hat{L}_{<1212>}^{0}+\tau_{<22 \geq}^{0}}{\vec{r}^{2}}
\end{aligned}
$$

The boundary conditions for the puckering problem are $\bar{w}=0$ on the flange, i.e. on the flat part of the shell held between the blank-holder and the die $\left(\bar{r} \equiv r+u(r)>R_{A}+R_{r}\right)$ while the edge $r=R_{L}$ remains traction free. The boundary conditions at the apex $r=0$ will be discussed later.

Any admissible functions $\vec{u}(r, \theta), \vec{v}(r, \theta), \bar{w}(r, \theta)$ can be put in the form

$$
\left.\begin{array}{c}
\bar{u}(r, \theta)=\sum_{n=0}^{\infty}\left[u_{n}^{\prime}(r) \cos (n \theta)+u_{n}(r) \sin (n \theta)\right], \\
\bar{v}(r, \theta)=\sum_{n=0}^{\infty}\left[v_{n}(r) \cos (n \theta)+v_{n}^{\prime}(r) \sin (n \theta)\right], \\
\bar{w}(r, \theta)=\sum_{n=0}^{\infty}\left[w_{n}^{\prime}(r) \cos (n \theta)+w_{n}(r) \sin (n \theta)\right] .
\end{array}\right\}
$$

Upon substitution of (3.21) into the bifurcation functional in (3.19) one obtains

$$
\begin{aligned}
F(\lambda ; \bar{u}, \bar{v}, \vec{w})=2 \pi\left[F_{0}\left(u_{0}^{\prime}, 0, w_{0}^{\prime}\right)+F_{0}\left(0, v_{0}, 0\right)\right] & \\
& +\pi \sum_{n=1}^{\infty}\left[F_{n}\left(u_{n}, v_{n}, w_{n}\right)+F_{n}\left(u_{n}^{\prime}, v_{n}^{\prime}, w_{n}^{\prime}\right)\right],
\end{aligned}
$$

with

$$
\begin{aligned}
& F_{n}(\lambda ; *, *, * * * *)=\int_{0}^{R_{L}}\left\{C_{11}\left[\left(\frac{\mathrm{d} u}{\mathrm{~d} r}+\lambda_{r} \bar{\kappa}_{r} *\right)^{2}+\frac{h^{2}}{12}\left(\frac{d}{\mathrm{~d} r}\left(\frac{1}{\lambda_{r}} \frac{\mathrm{d} w}{\mathrm{~d} r}\right)\right)^{2}\right]\right.
\end{aligned}
$$

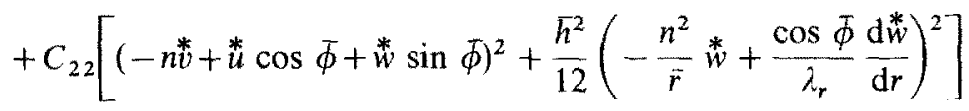

$$
\begin{aligned}
& +2 C_{12}\left[\left(\frac{\mathrm{d} u}{\mathrm{~d} r}+\lambda_{r} \bar{\kappa}_{r}^{*}\right)(-n v+* \operatorname{u} \cos \bar{\phi}+w \sin \bar{\phi})\right.
\end{aligned}
$$




$$
\begin{aligned}
& \left.+\frac{\hbar^{2}}{12}\left(\frac{d}{\mathrm{~d} r}\left(\frac{1}{\lambda_{r}} \frac{\mathrm{d} w}{\mathrm{~d} r}\right)\right)\left(-\frac{n^{2}}{\bar{r}} \stackrel{*}{w}+\frac{\cos \bar{\phi}}{\lambda_{r}} \frac{\mathrm{d}^{*}}{\mathrm{~d} r}\right)\right] \\
& +D_{11}\left[\left(\frac{\mathrm{d} v}{\mathrm{~d} r}\right)^{2}+\frac{h^{2}}{12}\left(\frac{d}{\mathrm{~d} r}\left(\frac{n w}{\bar{r}}\right)\right)^{2}\right]+D_{22}\left[\left(n *-{ }^{*} \cos \phi\right)^{2}\right.
\end{aligned}
$$

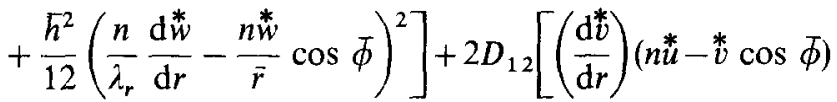

$$
\begin{aligned}
& \left.+\frac{h^{2}}{12}\left(\frac{d}{\mathrm{~d} r}\left(\frac{n *}{\bar{r}}\right)\right)\left(\frac{n}{\lambda_{r}} \frac{\mathrm{d} w^{*}}{\mathrm{~d} r}-\frac{n w}{\bar{r}} \cos \bar{\phi}\right)\right]+N_{r}\left(\frac{1}{\lambda_{r}} \frac{\mathrm{d} w^{*}}{\mathrm{~d} r}\right)^{2} \\
& \left.+N_{\theta}\left(\frac{n}{\bar{r}} \stackrel{*}{w}\right)^{2}\right\} \bar{r} \lambda_{r} \mathrm{~d} r
\end{aligned}
$$

The critical height $H_{c}=\lambda_{c}$ is then given by the minimum over all integers $n$ of the lowest eigenvalue $\lambda_{c}(n)$ for each $F_{n}$. The essential boundary conditions to be imposed on the

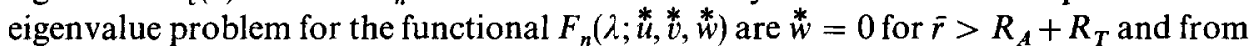
the finiteness of $F_{n}$, the apex conditions $\stackrel{*}{u}=\stackrel{*}{v}=\stackrel{*}{w}=\mathrm{d} \stackrel{*}{w} / \mathrm{d} r=0$ at $(r=0)$ for $n \neq 0$ and $u_{0}^{\prime}=0, \mathrm{~d} w_{0}^{\prime} / \mathrm{d} r=0, v_{0}=0$ for $n=0$.

At this point, it should be remarked that, in the formulation of the bifurcation functional $F$, the contact effects on the punch as well as on the die throat have been ignored. In reality assuming a Winkler type foundation the contact areas will depend on the unknown eigenmode, thus rendering the eigenvalue problem a nonlinear one. The functional $F_{n}$ in (3.23) will therefore provide a lower bound for the critical height $H_{c}$. An upper bound will be found if $F_{n}$ is augmented by the contact term $k w^{*} \mathbf{H}\left(R_{p}-d_{1}\right)+k w^{*} \mathbf{H}\left(R_{T}-d_{2}\right)$, where $k$ is the "foundation constant" (see (2.5), $(2.7))$.

\section{Numerical Method and Results}

\subsection{Finite element method}

The finite element method has been employed in order to evaluate the prebifurcation solution of the structure as well as the critical height $H_{c}(n)$ corresponding to a given wave number $n$.

The basis for the solution algorithm for the principal branch is the incremental virtual work formulation derived from (2.9), namely

$$
\Delta \lambda\left[(\text { I.V.W. })^{\cdot}-(\text { E.V.W. })^{\circ}\right]=-(\text { I.V.W. }- \text { E.V.W. }) .
$$

It can be seen that in the strain-displacement equations (2.3) and (2.4) (see also (2.6), (2.8)), only spatial derivatives up to first order of the total displacement components $u(r)$ and $w(r)$ are involved. Thus piecewise continuous $\left(C^{0}\right)$ shape functions will be employed here. Higher order shape functions cannot be used in this case in view of the anticipated 
slope discontinuities at the points of the loss of contact between the membrane and the punch or the die throat. Consequently the displacements $u(r)$ and $w(r)$ are linearly interpolated within each element.

Due to the expected rapid variations of the stress field as well as the loading direction angle $\theta$ in the neighborhood of the die throat, a power law type of refinement in the mesh has been adopted, namely

$$
\begin{aligned}
\left|r_{n}-\left(R_{A}+R_{T}\right)\right|=\Delta l\left|\frac{R_{A}+R_{T}+(\alpha-1) \Delta l-r_{0}}{\alpha \Delta l}\right|^{\alpha} ; \\
R_{A}+R_{T}+(2 \alpha-1) \Delta l>r_{0}>R_{A}+R_{T}-\Delta l,
\end{aligned}
$$

where $r_{n}$ is the new (refined) coordinate and $r_{0}$ the one corresponding to a uniform subdivision of the interval $\left[0, R_{L}+2(\alpha-1) \Delta l\right]$. The mesh refinement exponent is denoted by $\alpha(\alpha>1)$ and the refined zone of the plate extends by $\Delta l$ on each side of the circle with radius $R_{A}+R_{T}$. In all of the calculations reported here $\alpha=2$ and $\Delta l=3 R_{T}$. Some calculations were repeated with $\Delta l=5 R_{T}$ and $\alpha=3$ with the number of elements kept fixed, but no appreciable difference in the results was observed.

An incremental Newton--Raphson algorithm based on (4.1) has been adopted for the principal solution. In the case where the material is isotropic (transverse anisotropy ratio $R=1$ ) and the loading angle $\theta$ lies within the forward loading cone, the constitutive equation employed here is a path independent one (since the principal axes of stretch are fixed with respect to the material-see STÖREN and RICE (1975) for details) and steps of the order of the thickness $\Delta H=2 h$ or $4 h$ were found adequate for the convergence of the method with usually three or four iterations per load increment $\Delta H$. For the path dependent cases the step size used was much smaller, $\Delta H=0.1 \mathrm{~h}$, but in this case, only one iteration per step was required. The choice of the step size in the latter case was dictated by an $\mathrm{O}\left(10^{-3}\right)$ accuracy requirement on the stresses.

To avoid numerical problems at the beginning of the loading process, a slightly deformed stress free shape of the membrane was considered. More specifically, in the stress free state the initial vertical deviation $w_{0}(r)$ of a material point $r$ from the planar reference configuration is given by

$$
\begin{aligned}
& w_{0}=\left(R_{p}^{2}-r^{2}\right)^{1 / 2}-R_{p}+H_{0} \text { for } 0<r<R_{p} \sin \beta_{0} \text {, } \\
& \omega_{0}=R_{T}\left(1-\cos \beta_{0}\right)+\left[R_{A}+R_{T}\left(1-\sin \beta_{0}\right)-r\right] \tan \beta_{0} \\
& \left.\begin{array}{lll}
\quad \text { for } & R_{p} \sin \beta_{0}<r<R_{A}+R_{T}\left(1-\sin \beta_{0}\right), \\
w_{0}=R_{T}-\left(R_{T}^{2}-\left(R_{A}+R_{T}-r\right)^{2}\right)^{1 / 2} & \text { for } \quad R_{A}+R_{T}\left(1-\sin \beta_{0}\right)<r<R_{A}+R_{T}, \\
w_{0}=0 & \text { for } \quad R_{A}+R_{T}<r<R_{L},
\end{array}\right\}
\end{aligned}
$$

where $H_{0}$ is the distance of the apex of the membrane from the flat configuration and $2 \beta_{0}$ is the angle of contact between the punch and the membrane. The relation between $H_{0}$ and $\beta_{0}$ is found from simple geometrical considerations to be

$$
H_{0}=R_{p}+R_{T}+\left(\left(R_{A}+R_{T}\right) \sin \beta_{0}-\left(R_{p}+R_{T}\right)\right) / \cos \beta_{0}
$$


In the calculations reported here, $\beta_{0}$ was taken to be $\beta_{0}=0.035 \mathrm{rad}$. Some calculations have been repeated with $\beta_{0}=0.02$ and $\beta_{0}=0.05 \mathrm{rad}$ but no difference in the final stresses and critical punch displacements was observed.

The punch stiffness parameter $k$ was taken to be $k h / E=0.05 . \dagger$ It was found in this case that the penetration distances $d_{1}$ and $d_{2}$ (see (2.6) and (2.8)) never exceeded $10^{-2} h$.

Finally, the number of elements used in the calculations reported here depended on the initial radius of the plate $R_{L}$. The interval $\left[0, R_{L}+2(\alpha-1) \Delta l\right.$ was divided into equal subintervals of constant size $\Lambda r$. Most of the calculations were performed with $\Delta r=0.025 R_{A}$ although no significant difference in the results was observed from the case when a mesh with $\Delta r=0.05 R_{A}$ was used.

In order to find the critical height $H_{c}(n)$ for a given wave number $n$ the positive definiteness of the functional $F_{n}$ in (3.23) is examined at every step of the deformation process as the punch displacement $H$ increases. A finite element discretization of $F_{n}$ is employed using linear interpolation for $\stackrel{*}{u}$ and $v$ within each element and a hermitian one for $\stackrel{*}{*}$. The resulting discretized stiffness matrix, say $\mathbf{K}$, is subsequently decomposed using a modified Choelesky decomposition $\mathbf{K}=\mathbf{L D U}$, with $\mathbf{L}$ a lower triangular matrix, $\mathbf{U}=\mathbf{L}^{T}$ and $\mathbf{D}$ a diagonal matrix. Positive definiteness of $\mathbf{K}$ is lost as soon as an entry of $\mathbf{D}$ becomes negative. The results from the finite element calculations are reported below.

\subsection{Numerical results}

The first issue to be addressed is the influence of the "drawing ratio" $R_{L} / R_{A}$ on the shape of the first eigenmode, i.e. the relation between $R_{L} / R_{A}$ and the critical wavenumber $n_{c}$, corresponding to the critical punch displacement $H_{c}$ (see section 3 for the pertaining definition). The dependence of $n_{c}$ on $R_{L} / R_{A} t$ is depicted in Fig. 3. The corresponding calculations are performed for a transversely isotropic elastic-plastic material $(R=1)$, with hardening exponent $m=4$, total loading and elastic unloading cone angles $\theta_{0}=\pi / 2$ and $\theta_{c}=\pi$ respectively, for a punch angle $\phi=\pi / 3$ and an initial plate thickness $h=0.01 R_{A}$. The results show that the critical wavenumber increases with increasing drawing ratio and Fig. 3 is also a typical representative of all the $n_{c}-\left(R_{L} / R_{A}\right)$ curves obtained from the calculations reported subsequently. The trend for high values of the critical wavenumber is consistent with experimental evidence in puckering phenomena where a high number of circumferential ripples is observed on the unsupported part of the cup's wall as for example in SHILKRUT (1983) and the mild steel tests reported in the introduction (see Fig. 1). The trend for higher $n_{c}$ as $R_{L} / R_{A}$ increases can be explained from the observation that at bifurcation the width of the contact free annular zone of the plate decreases with increasing drawing ratio in view of the higher $H_{c}$. Since the characteristic wavelength in the circumferential direction is expected to be of the same order of magnitude as the one in the radial direction the resulting high value for $n_{\mathrm{c}}$ can then be easily understood.

\footnotetext{
$\dagger$ A higher and a lower value for $k$ have also been employed $(k h / E=0.02$ and 0.1$)$ but produced no appreciable difference in the resulting prebifurcation displacement and stress fields.

$\ddagger$ For some values of $R_{L} / R_{A}$ more than one critical wavenumbers $n_{c}$ are numerically found to correspond to approximately the same critical height $H_{c}$. The question, whether these eigenmodes are nearly or exactly simultaneous cannot, unfortunately, be provided by an approximate numerical method.
} 


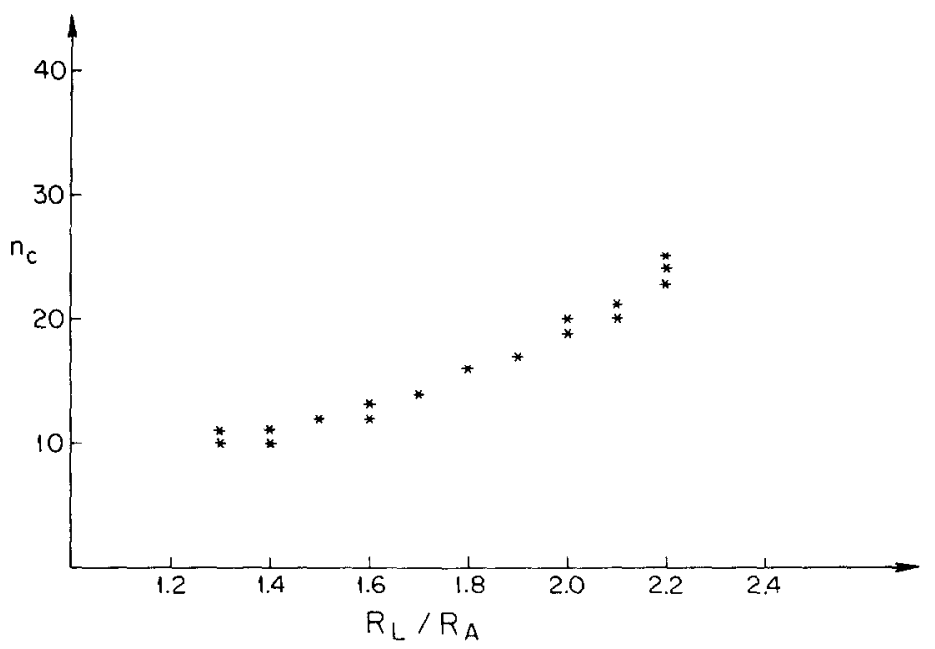

FIG. 3. Dependence of the critical wave number $n_{c}$ on the drawing ratio $R_{L} / R_{A}\left(\varepsilon_{y}=0.001, m=4, R=1\right.$, $\theta_{0}=\pi / 2, \theta_{c}=\pi, h=0.01 R_{A}, \phi=\pi / 3$ ).

The effects of the punch curvature on the puckering phenomenon are explored next. Figure 4 depicts the relation between the nondimensionalized critical punch displacement $H_{c} / R_{A}$ vs the drawing ratio $R_{L} / R_{A}$. All the calculations in this case are performed for the same material and plate thickness as before. The curve corresponding to the smallest punch curvature $\phi=\pi / 4$ stops at a drawing ratio of about 2.0 since for higher drawing ratios the spherical cup can be successfully drawn to its final shape without any puckering. For the two "sharper" punches, i.e. $\phi=\pi / 3$ and $\phi=\pi / 2$ calculations were stopped at values of the drawing ratio of about 2.2 since for higher values strain

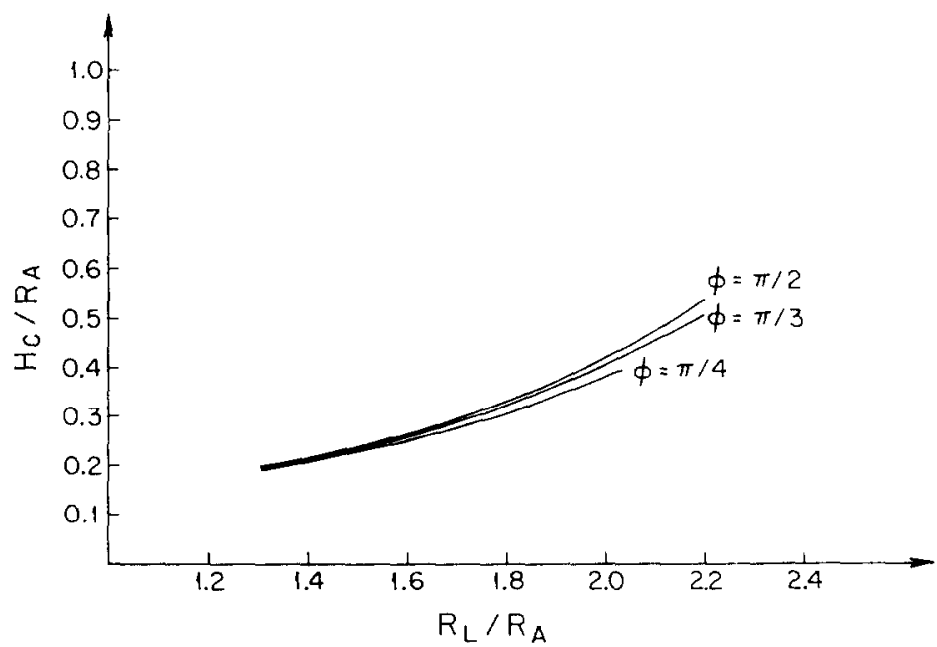

FIG. 4. Dependence of the nondimensionalized critical punch displacement $H_{\mathrm{c}} / R_{A}$ on the drawing ratio $R_{L} / R_{A}$ for different punch angles $\phi\left(\varepsilon_{y}=0.001, m=4, R=1, \theta_{0}=\pi / 2, \theta_{c}=\pi, h=0.01 R_{A}\right)$. 


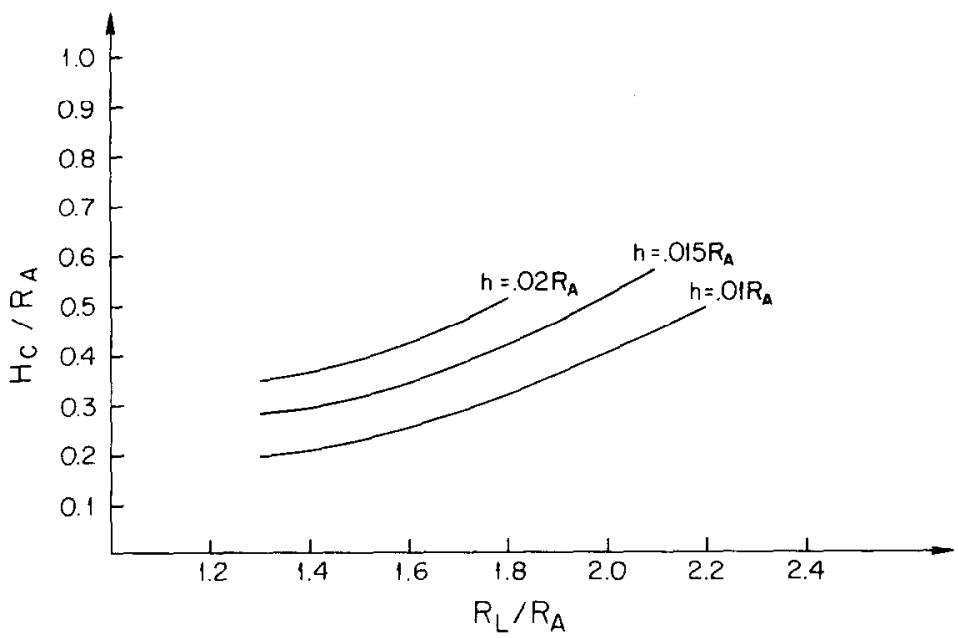

FIG. 5. Dependence of the nondimensionalized critical punch displacement $H_{c} / R_{A}$ on the drawing ratio $R_{L} / R_{A}$ for different plate thicknesses $h\left(\varepsilon_{y}=0.001, m=4, R=1, \theta_{0}=\pi / 2, \theta_{c}=\pi, \phi=\pi / 3\right)$.

localization in the principal (axisymmetric) solution precedes the onset of a nonaxisymmetric bifurcation. It is found that for a given material and initial plate geometry the critical height increases with increasing punch curvature. An explanation of this behaviour can be attributed to the fact that for a given punch displacement the compressive hoop stresses near the die throat are higher for the blunter punch and thus bifurcation for this case will precede the bifurcation for the sharper punch.

The effects of the plate's initial thickness on the critical punch displacement are investigated subsequently. Figure 5 depicts the relation between the nondimensionalized critical punch height $H_{c} / R_{A}$ vs the drawing ratio $R_{L} / R_{A}$ for a material with the same constitutive equation as in the previous calculations and for a punch angle $\phi=\pi / 3$. As expected the critical punch displacement increases significantly with increasing plate thickness (in view of the resulting increase of the plate's bending stiffness). Note that for $h / R_{A}=0.02,0.015$ the cup can be fully drawn without puckering for drawing ratios higher than approximately $R_{L} / R_{A}=1.8$ and 2.1 respectively, while for $h / R_{A}=0.01$ localization in the axisymmetric solutions occurs for $R_{L} / R_{A} \geqslant 2.2$ approximately.

The influence of material hardening on the critical punch displacement is examined in Fig. 6 for a transversely isotropic material with $R=1, \theta_{0}=\pi / 2, \theta_{c}=\pi$ and for hardening exponents $m=2,4,10$. The plate's initial thickness $h=0.01 R_{A}$ and the punch angle $\phi=\pi / 3$. The apparent paradox in Fig. 6 , where the softer the material is (i.e. the higher $m$ ), the more stable the plate becomes against puckering (i.e. $H_{c}$ increases), can be understood if one observes that, for a given plate geometry and punch height, the compressive hoop stresses are higher for the harder material.

All the aforementioned calculations were performed for a material with a forward loading cone angle $\theta_{0}=\pi / 2$ and, as the calculations show, the loading angle $\theta$ never exceeds $\theta_{0}$, thus making the above bifurcation calculations $J_{2}$ deformation theory of plasticity calculations, with a path independent principal branch. 


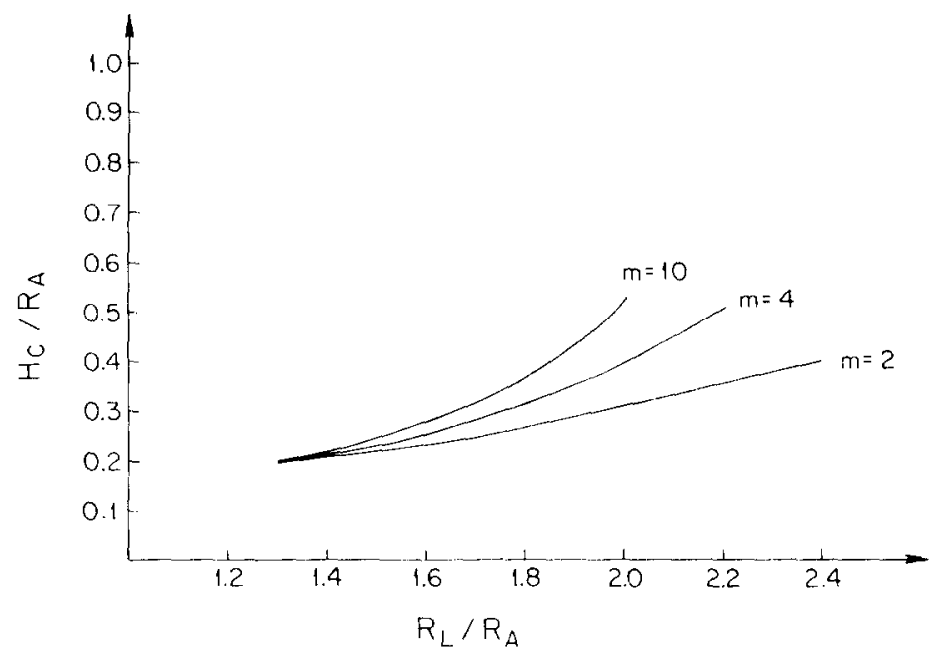

Fig. 6. Dependence of the nondimensionalized critical punch displacement $H_{c} / R_{A}$ on the drawing ratio $R_{L} / R_{A}$ for different hardening exponents $m\left(\varepsilon_{y}=0.001, R=1, \theta_{0}=\pi / 2, \theta_{\mathrm{c}}=\pi, h=0.01 R_{A}, \phi=\pi / 3\right)$.

The effects of using a path dependent corner type theory of plasticity in the calculations and the influence of the violation of the total loading condition in the principal branch of the solution, i.e. $\theta>\theta_{0}$ are investigated in the following.

In Fig. 7 is depicted the spatial variation of the loading direction angle $\theta$ for two materials having the same uniaxial stress-strain curve $m-4$ (also $R-1$ ) but different cone angle characteristics; in one material $\theta_{0}=0, \theta_{c}=3 \pi / 4$ while in the other $\theta_{0}=\pi / 4, \theta_{c}=3 \pi / 4$. Calculations are carried out for a plate of initial thickness

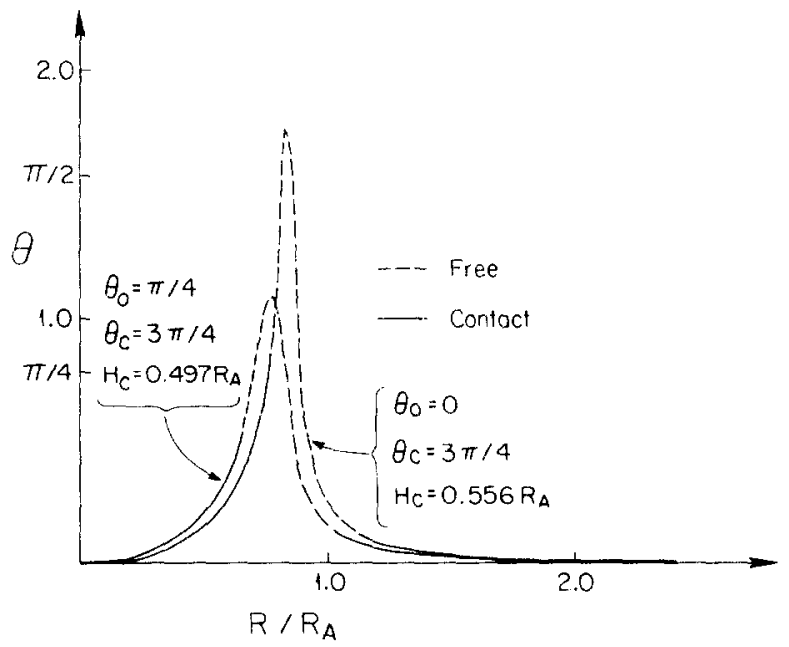

FIG. 7. Spatial distribution of the loading direction angle $\theta$ at the onset of bifurcation for two materials with different vertex characteristics $\left(\varepsilon_{y}=0.001, m=4, R=1, h=0.01 R_{A}, \phi=\pi / 3, R_{t} / R_{A}=2.2\right)$. 


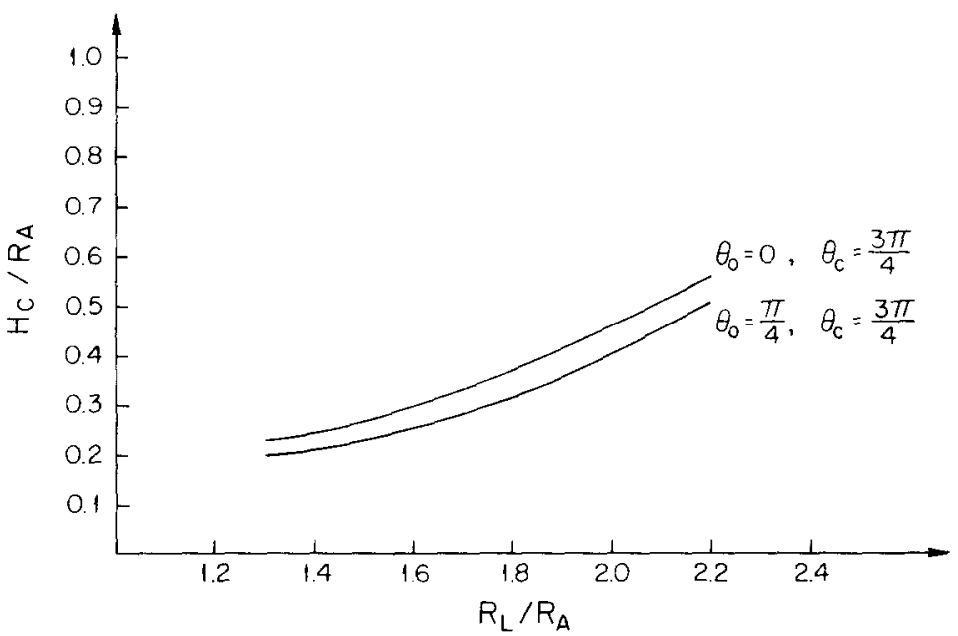

FiG. 8. Dependence of the nondimensionalized critical punch displacement $H_{\mathrm{r}} / R_{A}$ on the drawing ratio $R_{L} / R_{A}$ for two materials with different vertex characteristics but sharing the same geometry and remaining material properties $\left(\varepsilon_{y}=0.001, m=4, R=1, h=0.01 R_{A}, \phi=\pi / 3\right.$ ).

$h / R_{A}=0.01$, a punch angle $\phi=\pi / 3$ and aspect ratio $R_{L} / R_{A}=2.2$. As expected, in the contact free part of the plate (represented in dashed line in Fig. 7), material points undergo considerable deviations from proportional loading $(\theta=0$ corresponds to proportional loading). Note that the spatial distribution of $\theta$ depends strongly on the corner characteristics of the material; the smaller the total loading cone, the higher the maximum value of $\theta$.

The significant influence of the corner characteristics of the material on the prebifurcation solution and mainly on the loading direction angle $\theta$ will have a strong effect on the bifurcation calculations. In Fig. 8 the nondimensionalized critical height $H_{c} / R_{A}$ is plotted as a function of the drawing ratio $R_{L} / R_{A}$ for the two different materials employed in the calculations in Fig. 7. As expected, in view of the use of the current incremental moduli (evaluated on the principal branch of the solution) in the bifurcation fanctional (see (3.7) and also (3.23)), for a given plate and punch geometry bifurcation always occurs first in the plate with $\theta_{0}=\pi / 4, \theta_{\mathfrak{c}}=3 \pi / 4$ in view of the smaller (as compared with the $\theta_{0}=0, \theta_{c}=3 \pi / 4$ case) instantaneous moduli.

In all the above calculations the material is taken to be transversely isotropic $(R=1)$. Some of the calculations (more specifically the ones in Figs. 3 and 8 ) were repeated for $R$ values $0.5,1.5$ and 2.0 without any significant difference in the results (differences in $H_{c} / R_{A}$ are of the order of a few percent). This result is very similar in nature to the calculations of the effect of plastic anisotropy on flange wrinkling performed by TRIANTAFYLLIDIS and NEEDLEMAN (1980) where the transverse plastic anisotropy effect on the flange wrinkling was negligible in the case of a laterally unsupported flange.

Finally, in order to take into account the lateral constraint effects for the bifurcation eigenmode due to the presence of the punch and die throat, some of the calculations were repeated by modeling the contact areas as Winkler foundations in the way described at the end of Section 3. The differences found in $H_{c} / R_{A}$ were less than $10 \%$. At 
any rate the results presented here are lower bounds for the critical punch displacements with rather small differences from the upper bounds found from the aforementioned calculations.

\section{Concluding Remarks}

The preceding analysis of the puckering instability in the hemispherical cup test shows the following trends on the main geometrical and material parameters governing this phenomenon.

The critical height of the punch is an increasing function of the drawing ratio and thus a larger flange has a delaying effect on the puckering instability. This is exactly the opposite of what happens in the wrinkling case of a flat flange (see TRIANTAFYLLIDIS and NeEDleman, 1980) where the wider the flange the earlier the plastic bifurcation occurs. Contrary to what one might naively expect, the harder the material the more prone it is to puckering; this trend is again the opposite of what happens in the wrinkling problem. In addition, and consistently with experimental observations, the critical wavenumber at the onset of bifurcation is considerably higher than the one corresponding to the wrinkling of a flange with similar geometric and material properties.

In view of the membrane approximation (adopted for the resulting simplicity in the formulation of the problem) only fairly weak curvatures of the resulting deformed shell can be investigated $\left(h / R_{p}<0.02\right.$ here). Consequently, the effect of the punch angle $\phi$ is not found to be important.

The compressive hoop stresses in the unsupported wall of the deformed cup, which are responsible for the plastic instability, are adequately smaller than the maximal stresses which occur near the apex, and thus relatively unaffected by the changes in the transverse plastic anisotropy. Therefore only minor changes in the critical height are found for different plastic anisotropy ratios. Similar results were found for the laterally unconstrained wrinkling of a flat flange by TRIAN rAFYLLIDIS and NEEDLEMAN (1980).

The phenomenological corner theory employed in these calculations is an attractive alternative to the simpler deformation theory models (usually employed in plastic stability calculations) in view of the large deviations of the prebifurcation solution from proportional loading. Calculations show significant influence of the (unfortunately experimentally nonverifiable) corner characteristics of the theory. The strong deviations from proportional loading are found to have an important stiffening effect in the material and thus the onset of plastic buckling occurs at values considerably higher than those predicted by simpler deformation theories. Although qualitatively correct, the quantitative predictions of the model should be regarded as suggestive. More complicated constitutive models can be used in this direction with considerable penalty, however, in the complexity of the calculations. One such example might be a rate sensitive constitutive model in conjunction with a nonaxisymmetrically imperfect plate. A kinematically hardening nonaxisymmetrically imperfect plate is another possibility. Such modelizations appear to be the evident next step (in conjunction with some careful experiments) for the analysis of this problem, especially when the postbifurcation behaviour of the plate is also of interest. 


\section{ACKNOWLEDGEMENTS}

Support for this work by the National Science Foundation under Contract NSF-MEA 8116449 is gratefully acknowledged. The author would also like to thank Dr. S. K. Samanta from the Scientific Research Laboratories of Ford Motor Company for interesting discussions and for providing the puckered specimen in Fig. 1.

\section{REFFRFNCES}

Christoffersen, J. and HUTCHINSON, J. W.

Chu, C. C.

Devons, J. D,

HeCKER, S. S.

HiLl, $\mathbf{R}$.

HILL, R.

HUTCHINSON, J. W.

HuTCHINSON, J. W.

LIN, T. H.

SHILKRUT, D. 1.

STÖREN, S, and RICE, J. R.

TRIANTAFYLLIDIS, $N$. and NeEdleman, $A$.

TRIANTAFYLLIDIS, $N$. NeEdleman, $A$. and

TVERGAARD, $\mathrm{V}$.

Triantafyllidis, $N$.

TVERGaARd, V., NeEdleman, A. and Lo, K. K.

TVERGAARD, $\mathrm{V}$.

WANG, N. and BUDIANSKY, B.
1979 J. Mech. Phys. Solids 27, 465.

1980 Int. J. Solids Structures 16, 913.

1941 The Metallurgy of Deep Drawing and Pressing, Chapman Hall, London.

1976 Constitutive Equations in Viscoplasticity: Computational and Engineering Aspects. $A M D$ 20, ASME, New York.

1958 J. Mech. Phys. Solids 6, 236.

1967 J. Mech. Phys. Solids 15, 79.

1970 Proc. R. Soc. Lond. A 319, p. 247.

1973 J. Mech. Phys. Solids 21, 163.

1974 Adv. Appl. Mech. 14, 67.

1971 Adv. Appl. Mech.11, 255.

1983 J. Engng Mech. 109, 289.

1975 J. Mech. Phys. Solids 23, 421.

1980 J. Engng Matls Technol. 102, 241.

1982 Int. J. Solids Structures 18, 129.

1983 J. Mech. Phys. Solids 31, 499.

$1981 J$ J.Mech. Phys. Solids 29, 115.

1982 D.C.A.A.M. Report 4, No. 250.

1978 J. Appl. Mech. 45, 73. 